\title{
A hybrid approach to multi-depot multiple traveling salesman problem based on firefly algorithm and ant colony optimization
}

\author{
Olief Ilmandira Ratu Farisi ${ }^{1}$, Budi Setiyono ${ }^{2}$, R. Imbang Danandjojo ${ }^{3}$ \\ ${ }^{1}$ Department of Mathematics Education, University of Nurul Jadid, Probolinggo, Indonesia \\ ${ }^{2}$ Department of Mathematics, Institut Teknologi Sepuluh Nopember, Surabaya, Indonesia \\ ${ }^{3}$ Research and Development Agency of Transportation, Ministry of Transportation Republic of Indonesia, Jakarta, \\ Indonesia
}

\begin{tabular}{l}
\hline \hline Article Info \\
\hline Article history: \\
Received Feb 20, 2021 \\
Revised Aug 25, 2021 \\
Accepted Sep 12, 2021 \\
\hline
\end{tabular}

Keywords:

Ant colony optimization

Firefly algorithm

Multi-depot multiple traveling

salesman problem

\begin{abstract}
This study proposed a hybrid approach of firefly algorithm (FA) and ant colony optimization (ACO) for solving multi-depot multiple traveling salesman problem, a TSP with more than one salesman and departure city. The FA is fast converging but easily trapped into the local optimum. The ACO has a great ability to search for the solution but it converges slowly. To get a better result and convergence time, we integrate FA to find the local solutions and ACO to find a global solution. The local solutions of the FA are normalized then initialized to the quantity of pheromones for running the ACO. Furthermore, we experimented with the best parameters in order to optimize the solution. In justification, we used the sea transportation route in Indonesia as a case study. The experimental results showed that the hybrid approach of FA and ACO has superior performance with an average computational time of $26.90 \%$ and converges $32.75 \%$ faster than ACO.
\end{abstract}

This is an open access article under the CC BY-SA license.

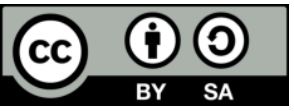

\section{Corresponding Author:}

Olief Ilmandira Ratu Farisi

Department of Mathematics Education

University of Nurul Jadid

PP. Nurul Jadid, Karanganyar, Paiton Probolinggo 67291, Indonesia

Email: farisi@unuja.ac.id

\section{INTRODUCTION}

Multi-depot multiple traveling salesman problem (MmTSP) is the shortest route problem which is the further development of traveling salesman problem (TSP). In MmTSP, more than one salesman must visit a set of cities exactly once and depart from different departure cities, called depots, and must return to their respective departure cities [1]. Finding the exact solution of the shortest route is more difficult as the number of visited cities increase [2].

Unlike TSP which has been extensively researched recently years, the MmTSP is not intensively studied. In fact, many applications which are related to routing problems with multi-depot can be modeled as MmTSP, such as the logistic distribution problem [3], vehicle routing problem [4], vehicle scheduling problem [5], split-delivery vehicle routing problem [6], and the location routing problem [7], [8]. Since TSP is NP-hard and the MmTSP includes the TSP as a particular case, so it has a higher complexity than TSP [9]. Therefore, it would be very useful to design an algorithm that has a good performance and produce a better solution of MmTSP.

Several researchers have conducted studies on the heuristic algorithms for solving MmTSP, such as multiple depots and closed paths (MDCP) method [10], genetic algorithm (GA) [11], firefly algorithm (FA) [12], and ant colony optimization (ACO) [13], [14]. According to Ghafurian and Javadian [13], the ACO is 
compared to Lingo 8.0 and showed that the ACO obtained an optimal solution faster than Lingo 8.0. The ACO is an optimization algorithm that adopts the behavior of ant colonies which was developed by Dorigo to solve TSP [15]. The ACO is robust and has a great ability in finding NP-hard problems solution [16]. It is also easily applied and understandable [17]. However, the change in the ACO's probability distribution in each iteration causes the uncertainty of the ACO's convergence [18]. Therefore, a few researchers developed a hybrid approach to improve the robustness of the ACO method, such as the integration of FA and ACO.

The FA is an algorithm based on the flashing behaviour of fireflies and was proposed by Yang [19]. The FA is easy to implement and has a fast convergence capability but it can be trapped into local optimum solution [20], [21]. A combination of FA and ACO approaches has been developed in solving various problems, such as economic power dispatch [22], traveling salesman problem (TSP) [23], and constrained engineering design problems [24]. According to the researches, a hybrid approach of FA-ACO has better performance than ACO itself.

In this regard to improve the accuracy and convergence time of ACO in finding the solution of MmTSP, we propose a hybrid approach of FA and ACO to optimize sea transportation routes in Indonesia to increase the effectiveness and efficiency of sea transportation services by the ministry of transportation. This problem can be modeled as a MmTSP since 51 ports must be visited with 5 ports being the main focus of the sea highway development as depots. Using the fast converge of FA, we find the local solutions with a shorter time. The local solutions are used for pheromone's initialization in the ACO to find the optimal solution.

\section{PROPOSED METHOD}

In this paper, we develop a hybrid FA-ACO to solve MmTSP. In this method, FA is used to find the local solutions then ACO will find the global solution based on FA's solutions. By finding the best combination of the lower possible number of ants and fireflies as well as all the parameters needed to run FA and $\mathrm{ACO}$, we can optimize the solution and minimize the convergence time. In the ACO method, the initial pheromones trail is distributed equally for each edge. But, in the hybrid FA-ACO, we set the initial trail based on the local solutions obtained by FA. The pheromones will be added on the edge which is part of the best local solutions. In this way, the probability of ants visiting the edge is bigger. The stages of the proposed method are shown in Figure 1.

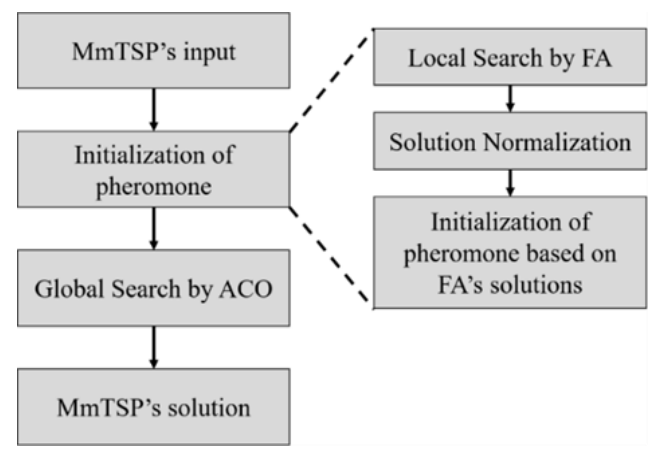

Figure 1. Stages of hybrid firefly algorithm-ant colony optimization

\subsection{Local search by FA}

Firefly Algorithm is an algorithm that is inspired by light-based fireflies' movements. It was first introduced by Yang [19] to solve continuous problems. To solve the permutation problem like MmTSP, the FA is discretized [25].

Fireflies move towards other fireflies that have a brighter light. The intensity of the light is influenced by the distance and absorption of light by air. The farther the distance, the smaller the intensity of the light. Also, the greater the absorption of light, the smaller the intensity of the light produced.

Based on [26] work, in this study, the fireflies represent permutation solutions of MmTSP. For example, Figure 2 shows the permutation representation of MmTSP with city- 1 and city- 5 as depots. The tour for the first salesman is 1-8-4-10-6-1 while the second salesman's tour is 5-7-9-3-2-5.

Since the objective function of the MmTSP is minimizing the cost, the light intensity of a firefly $\left(I_{k}\right)$ is calculated as the inverse of the total cost. The smaller the total cost produced, the brighter the light intensity emitted. After the fireflies have their brightness, the distance between two fireflies is determined by (1), 


$$
d(i, j)=\frac{\Delta d(i, j)}{N} \times 10
$$

where $\Delta d(i, j)$ is the number of different edges between the permutation representation of firefly- $i$ and firefly- $j$, and $N$ is the number of cities. In Figure 3, three edges 4-10,10-6,7-9 in firefly- $i$ do not belong in firefly-j. In other words, there are three edges 4-6, 7-10, 10-9 in firefly-j do not exist in firefly- $i$. Therefore, the distance between firefly- $i$ and firefly-j is 3 . A firefly moves toward the most attractive firefly based on the brightness and the distance. The attractiveness of firefly- $j$ seen by firefly- $i$ is any monotonic decreasing function in (2),

$$
\xi(i, j)=\xi_{0} e^{-\gamma d^{2}}
$$

where $d$ is the distance between the two fireflies, $\xi_{0}$ is the light intensity of firefly-j at zero distance, and $\gamma \in$ $[0, \infty)$ is light absorption. If $\gamma \rightarrow 0$, then $\xi(i, j)=\xi_{0}$. It means that at any distance, the attractiveness of firefly- $j$ when seen by firefly- $i$ is the same. If $\gamma \rightarrow \infty$, then firefly- $j$ cannot be seen at any distance because the value of light absorption is too large. Hence, the value of $\gamma$ must be tested because it will affect on the attractiveness of fireflies. The coefficient $\gamma$ is in the interval [0.01, 0.15].

A firefly moves toward the other firefly that has the most attractiveness. If there are no fireflies with greater attractiveness, it moves randomly. The number of movement's steps that must be taken by firefly- $i$ which attracted to the brighter firefly- $j$ is (3);

$$
x_{i}=\operatorname{random}(1, \Delta d(i, j))
$$

In MmTSP, the movement of firefly is the inversion mutation of its permutation representation. Inverse mutations are carried out in visited cities excluding depots. By using inversion mutation, the previous permutation represented by the firefly is not deformed. Figure 4 illustrate the movement of firefly- $i$.

This movement will make changes to the solutions represented by fireflies. Since each firefly moves

\begin{tabular}{|c|c|c|c|c|c|c|c|c|c|c|c|}
\hline 1 & 8 & 4 & 10 & 6 & 1 & 5 & 7 & 9 & 3 & 2 & 5 \\
\hline
\end{tabular}
for $m$ times, it will produce $m$ new solutions. If there are $p$ moving fireflies, there will be $p m$ new solutions. Choose the best solutions $p$ of $p+p m$ existing solutions as the new population of fireflies for the next iteration.

Figure 2. Permutation representation of MmTSP's solution

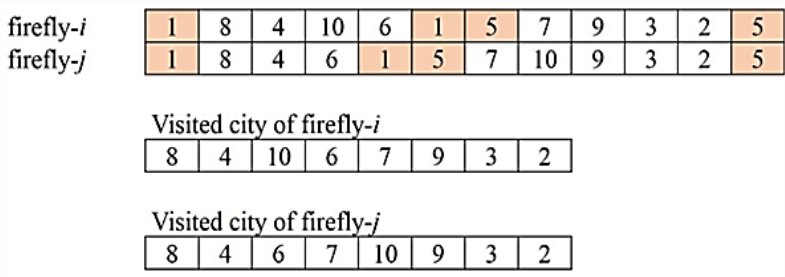

Figure 3. The distance of the two fireflies is the different edges between firefly-i and firefly-j

Visited city of firefly- $i$
\begin{tabular}{|c|c|c|c|c|c|c|c|}
\hline 8 & 4 & 10 & 6 & 7 & 9 & 3 & 2 \\
\hline
\end{tabular}
\begin{tabular}{|l|c|c|c|c|c|c|c|}
\hline Visited city of firefly- $i$ \\
\hline 8 & 4 & 7 & 6 & 10 & 9 & 3 & 2 \\
\hline
\end{tabular}

Figure 4. Inversion mutation with 2 length movements

\subsection{Normalization and initialization}

After FA satisfied the stopping condition, there will be $p$ best fireflies. In several cases, there is a possibility that some of the fireflies have the same representation of the solution. Hence, it is necessary to normalize the solutions. The same solutions will be considered as one solution. Then, assume that there are $q$ solutions as candidate tours after normalization. From the $q$ candidate tours, add the pheromones on the edges included in the tours to initialize the pheromones of ACO. The rules for adding pheromones are based on the order of the best solutions which is defined as (4); 


$$
\Delta t_{i, j}=\frac{10}{q}(q-k+1)
$$

where $(i, j)$ is the edge of the $k$-th best solution of the $q$ existing solutions. In this way, the edge that is part of the first-best solution will get more pheromones than the edges on the second-best solution. Thus, the most passed edges of the $q$ candidate tours will get the most pheromones addition. By setting initial pheromones with different quantity based on the local solutions of the FA, it will minimize the number of agents and iterations for running the ACO.

\subsection{Global search by ACO}

ACO is an algorithm developed by Dorigo to find the shortest path which adapts the behavior of the ants [15]. In finding food sources, ants produce pheromones which is useful for recognizing same-sex, other individuals, and groups as well as for helping the reproductive process. Moving ants will release pheromones on the ground and create a trail. After a certain interval, pheromones will experience evaporation.

For finding the global solution of MmTSP, place $n$ ants at one of the depots. For each ant, the first element of the tabu list is equal to its depot. Set initial trail $\tau_{i j}(t)$ for quantity of pheromones at time $t$ on edge $(i, j)$ from normalization results based on the FA's solutions. The probability of ant- $k$ moves from city- $i$ to city- $j$ is defined as (5):

$$
p_{i j}=\left\{\begin{array}{c}
\frac{\left[\tau_{i j}(t)\right]^{\alpha}\left[\eta_{i j}(t)\right]^{\beta}}{\sum u \in U_{k}\left[\tau_{i u}(t)\right]^{\alpha}\left[\eta_{i u}(t)\right]^{\beta}} \\
0, \text { otherwise }
\end{array}\right.
$$

where $\eta_{i j}$ is the visibility namely the inverse of the total distance, and $U_{k}$ is the set of unvisited cities by ant- $k$, with $\alpha$ and $\beta$ are the factors that influences the pheromones and visibility, respectively. After all of the ants have completed a tour, the tabu list is filled with the visited cities produced by each ant. Then, the trail intensity is updated using the global pheromones update rule.

The pheromones update rule involves the evaporation on all edges and pheromones' addition on the ants' passed edges. It is also affected by the distance produced by the ants. The shorter the tour, the more pheromones the ants leave behind. Thus, the edges with lots of pheromones will be more desirable on tours in the next iterations. The global pheromones update rules defined as (6):

$$
\tau_{i j}(\text { new })=(1-\rho) \tau_{i j}(\text { old })+\sum_{k=1}^{m} \Delta \tau_{i j}^{k}
$$

where $\rho$ represents the evaporation of trail, and $\Delta \tau_{i j}^{k}$ is the addition of pheromones produced by ant- $k$ on the edge $(i, j)$ is calculated by $(7)$,

$$
\Delta \tau_{i j}^{k}=\left\{\begin{array}{c}
\frac{Q}{d_{k}}, \text { if ant }-k \text { uses }(i, j) \\
0, \text { otherwise }
\end{array}\right.
$$

where $Q$ is a constant and $d_{k}$ is the total distance passed of ant- $k$.

The best tour produced by the ants is saved and the tabu list is emptied to save the tour for the next iteration. This process will be repeated until it meets a stopping condition. At the end of the method, we will obtain the tour with minimum cost as the solution of MmTSP.

\section{RESULT AND DISCUSSION}

\subsection{Mathematical Modeling}

Based on the mathematical model of MmTSP [27], the sea transportation route problem in Indonesia can be modeled as follows. Let $N$ be a finite set of ports and $C_{i j}=\left[c_{i j}\right],(i, j \in N)$ be the cost matrix from port- $i$ to port-j. The set $N$ is partitioned into $N=D \cup N^{\prime}$ where $D$ be the first five-port of $N$ as depots and $N^{\prime}=$ $\{6,7, \ldots, 51\}$ be the set of ports excluding depots. There is $1_{i}$ ship is placed at depot- $i$.

For any ships, $v_{i}$ is the number of visited ports on the ship's path from depot to port- $i$. Constant $L$ and $U$ are the minimum and the maximum number of ports a ship may visit, respectively; thus, $1 \leq v_{i} \leq$ $U \forall i>2$. In this study, we used lower bound $L=8$ and upper bound $U=12$. A binary variable $x_{i j k}$ is defined as equal to 1 if the ship departure from the $k$ th port passes the $(i, j)$ and $x_{i j k}$ is equal to 0 , otherwise. If $x_{i k k}=$ 1 , then $8 \leq v_{i} \leq 11$ must be satisfied; 


$$
\min \left(\sum_{k \in D} \sum_{j \in N^{\prime}}\left(c_{k j} x_{k j k}+c_{j k} x_{j k k}\right)+\sum_{k \in D} \sum_{i \in N^{\prime}} \sum_{j \in N^{\prime}} c_{i j} x_{i j k}\right)
$$

S.t

$$
\begin{aligned}
& \sum_{j \in N^{\prime}} x_{k j k}=1_{k}, k \in D \\
& \sum_{k \in D} x_{k j k}+\sum_{k \in D} \sum_{i \in N^{\prime}} x_{i j k}=1, j \in N^{\prime} \\
& x_{k j k}+\sum_{i \in N^{\prime}} x_{i j k}-x_{j k k}-\sum_{i \in N^{\prime}} x_{j i k}=0, k \in D, j \in N^{\prime} \\
& \sum_{j \in N^{\prime}} x_{k j k}-\sum_{j \in N^{\prime}} x_{j k k}=0, k \in D^{\prime} \\
& v_{i}+10 \sum_{k \in D} x_{k i k}+\sum_{k \in D} x_{i k k} \leq 11, i \in N^{\prime} \\
& v_{i}+\sum_{k \in D} x_{k i k}+(-6) \sum_{k \in D} x_{i k k} \leq 2, i \in N^{\prime} \\
& \sum_{k \in D} x_{k i k}+\sum_{k \in D} x_{i k k} \leq 1, i \in N^{\prime} \\
& v_{i}-v_{j}+12 \sum_{k \in D} x_{i j k}+10 \sum_{k \in D} x_{j i k} \leq 11, i \neq j ; i, j \in N^{\prime}
\end{aligned}
$$

From the formulation, (8) is the objective function. As shown in (9) ensures that there is exactly $1_{k}$ ship depart from each depot. As shown in (10) ensures that each port is visited exactly once. As shown in (11) and (12) are constraints that represent route continuity for ports and depots, respectively. Inequality (13) and (14) require upper and lower bound to the number of ports in the tour, respectively. These constraints $v_{i}$ to be equal to 1 , if $i$ is the first port in a tour. Inequality (15) prohibits tours with just one port. Inequality (16) is sub-tour elimination constraints (SECs) that prevent possible solutions which have closed tours of ports with no depots as starting and ending ports.

\subsection{Experimental results}

The experiment is implemented on a $2.2 \mathrm{GHz}$ Intel(R) Core(TM) i7 computer with 4.00 GB RAM using MATLAB. The experiment is examined for the sea transportation route. There are 51 ports with 5 ports as depots. In this study, there is exactly one ship in each depot and each ship may only visit a minimum of 8 ports as lower bound and a maximum of 12 ports as the upper bound.

We conducted an experiment to find the best parameters for running a local search by the FA and a global search by the ACO. The parameters play an important role in finding a solution, as shown in Figure 5. The parameters used are those that can reach the optimal solution in the least possible time. The minimum number of iterations to run a local search by FA is 100 iterations shown in Figure 5(a). For running a global search by the ACO, the convergence is reached after 229 iterations shown in Figure 5(a). The result is obtained by running the method for 1000 iterations. If no improvement of the result after 100 successive iterations, then we stop the iterations. In Figure 5(b) and Figure 5(c), 4 fireflies and 5 movements for each firefly, respectively, are enough to get the optimum solution. Figure 5(d) indicated the value of light absorption to get an optimal solution. We get $\gamma=0.11$.

Table 1 shows the correlation of $\alpha$ and $\beta$ to get an optimum solution. The value tested were: $\alpha=$ $\{0.5,1,2\}$ and $\beta=\{1,2,5\}$. The optimal solution is reached when $\alpha=1$ and $\beta=5$. We used these values for each experiment. In Figure 5(e), the best solution is obtained when $\rho=0.5$. The minimum number of ants used for global search is 20 shown in Figure 5(f). 


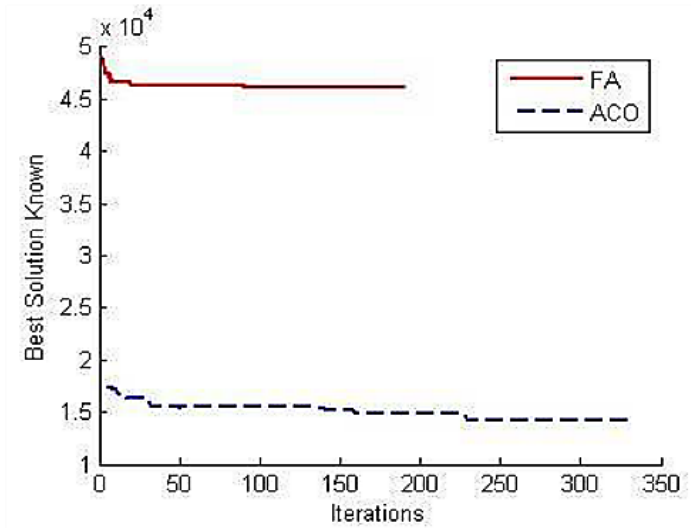

(a)

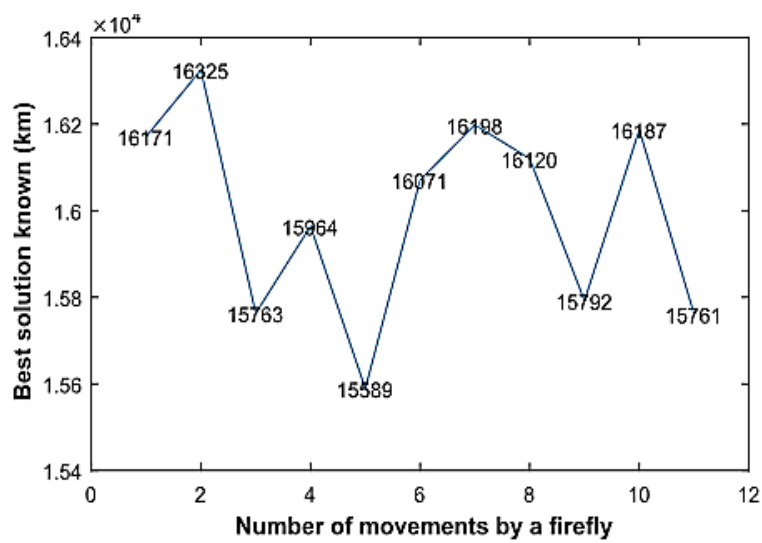

(c)

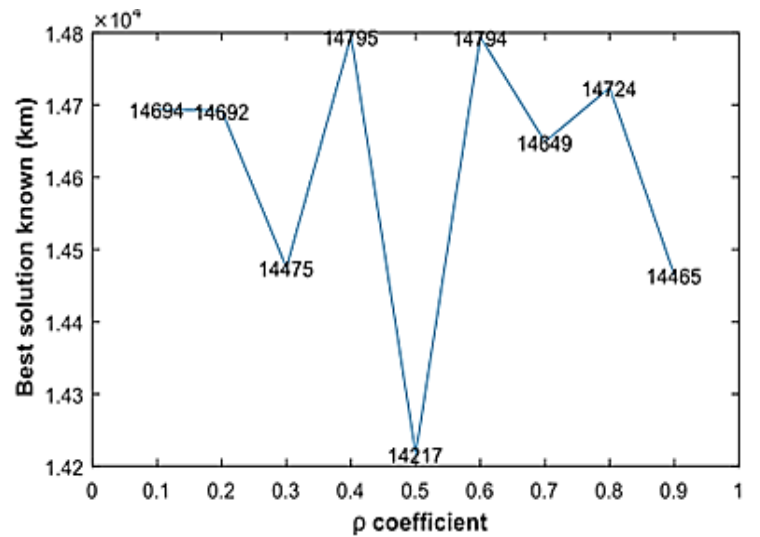

(e)

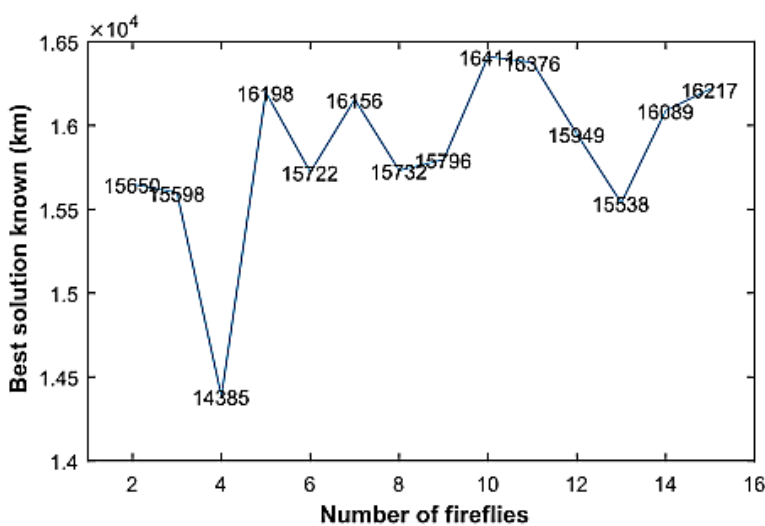

(b)

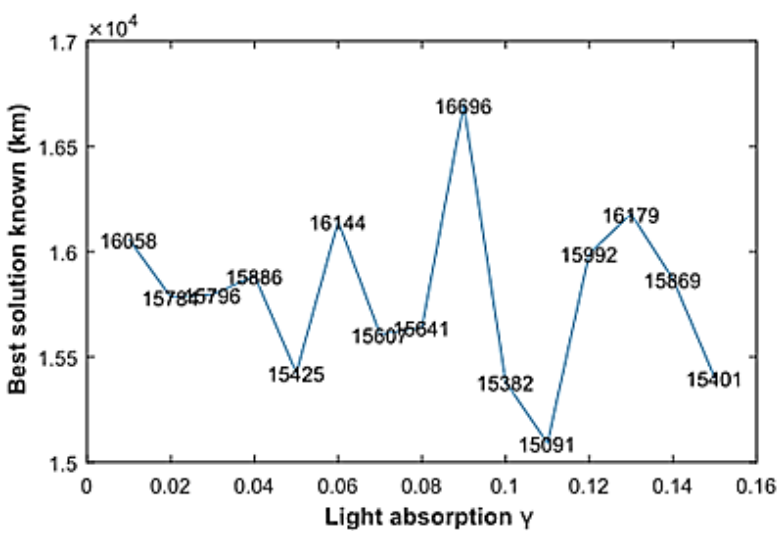

(d)

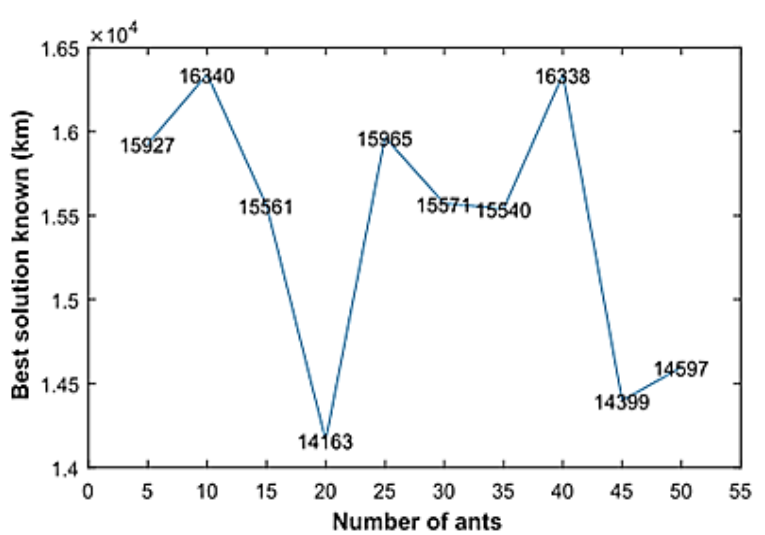

(f)

Figure 5. Parameter correlation with the best solution known, (a) the number of minimum iterations needed by FA local search and ACO global search, (b) correlation of the number of fireflies with the best solution known, (c) correlation of the number of movements with the best solution known, (d) correlation of light absorption with the best solution known, (e) correlation of coefficient with the best solution known,

(f) correlation of the number of ants with the best solution known

From the experiments, the parameter settings to execute hybrid FA-ACO are $\gamma=0.11, \alpha=1, \beta=5, \rho=0.5, Q=100,4$ fireflies with 5 movements, 20 ants, and 100 and 229 iterations of the FA and ACO, respectively. As shown in Figure 6 the comparison of performance between hybrid FA-ACO and ACO, Figure 6(a) presents the solution of hybrid FA-ACO and ACO after running the experiment 50 times. The hybrid FA-ACO reached the best solutions 31 out of 50 times by achieving an optimal solution that is better than ACO. It showed that the hybrid FA-ACO has better performance than ACO. 
The performance's comparison of hybrid FA-ACO and ACO from 50 experiments is shown in Table 2. Our proposed method achieved the best solution known closer to the exact solution than ACO. The hybrid FA-ACO has an average solution better than the ACO. The average of convergence time of the method is also better than ACO. It can conclude that the proposed method yields a better solution and computationally faster than ACO.

Figure 6(b) shows the convergence of hybrid FA-ACO and ACO to achieve the best solution. In getting the best solution, which is $13828 \mathrm{~km}$, the hybrid FA-ACO converged after 16.86102 seconds. Meanwhile, the convergence of ACO whose best solution is $13882 \mathrm{~km} \mathrm{~km}$ reached after 25.07218 seconds these results proved that our proposed method can improve the performance of ACO. It can find a better solution and converge faster than the ACO.

Table 1. Correlation of and coefficient with the best solution known

\begin{tabular}{cccc}
\hline$\alpha$ & $\beta$ & The best solution is known $(\mathrm{km})$ & Running time $(\mathrm{s})$ \\
\hline 0.5 & 1.0 & 21038 & 81.4154 \\
0.5 & 2.0 & 17169 & 80.0412 \\
0.5 & 5.0 & 14659 & 83.1577 \\
1.0 & 1.0 & 19088 & 80.0881 \\
1.0 & 2.0 & 16072 & 79.0493 \\
1.0 & 5.0 & 14231 & 81.7190 \\
2.0 & 1.0 & 18872 & 79.8144 \\
2.0 & 2.0 & 16532 & 77.6645 \\
2.0 & 5.0 & 15361 & 81.1222 \\
\hline
\end{tabular}

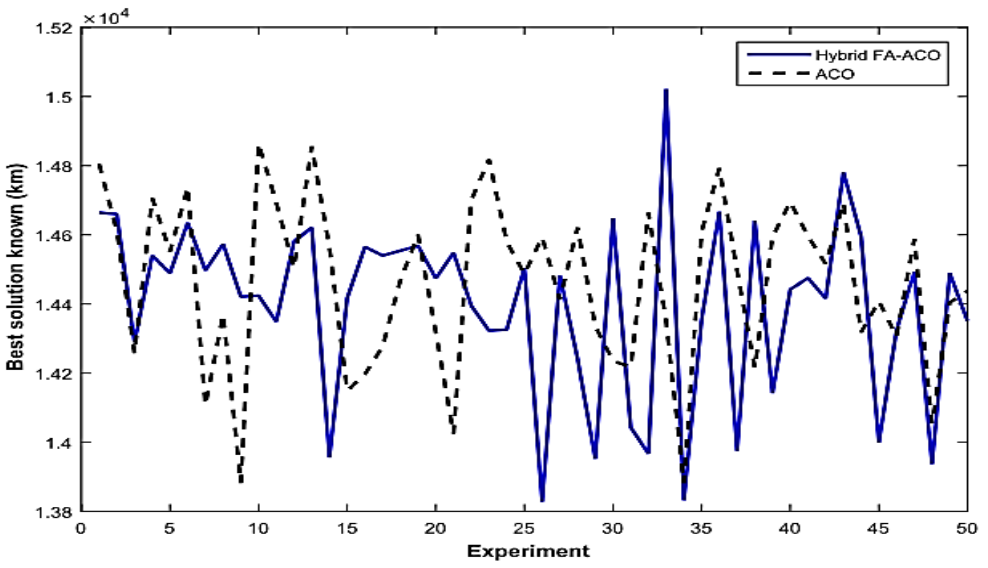

(a)

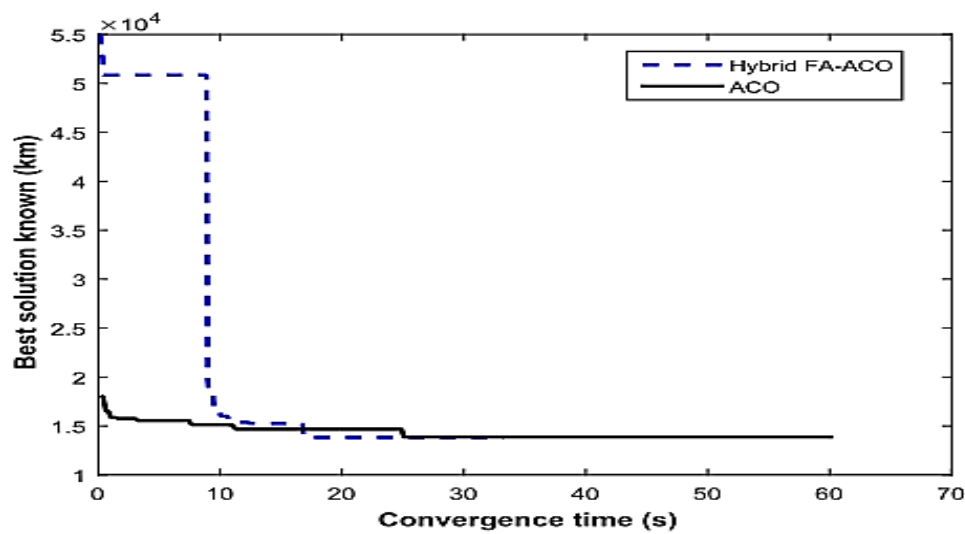

(b)

Figure 6. The comparison of performance between hybrid FA-ACO and ACO; (a) in reaching the best solution known from 50 experiments, (b) convergence time in reaching the best solution 
Table 2. Comparisons of hybrid FA-ACO and ACO from 50 experiments

\begin{tabular}{cccc}
\hline Method & The best solution is known $(\mathrm{km})$ & Average solution $(\mathrm{km})$ & Average time $(\mathrm{s})$ \\
\hline Hybrid FA-ACO & 13828 & 14400.28 & 44.42685 \\
ACO & 13882 & 14463.24 & 60.77814 \\
\hline
\end{tabular}

\section{CONCLUSION}

In this paper, a hybrid approach based on FA and ACO for solving multi-depot multiple traveling salesman problems was presented. The proposed method has three main stages: i) local search by FA; ii) normalization and initialization; and iii) global search by ACO. The main purpose is to find a better solution and minimize the time of convergence. Parameters play an important role in running the hybrid FA-ACO. The best parameter settings to execute hybrid FA-ACO to solve 51 ports with 5 depots and one salesman in each depot are $\gamma=0.11, \alpha=1, \beta=5, \rho=0.5, Q=100,4$ fireflies with 5 movements, 20 ants, and 100 and 229 iterations of the FA and ACO, respectively. The experiment results demonstrated that the hybrid FAACO provides better performance than the ACO. Our method obtained the best solution better than ACO and achieved an average computation time of $26.90 \%$ faster than ACO. Also, to reach the best solution, hybrid FA-ACO converges $32.75 \%$ faster than ACO.

\section{REFERENCES}

[1] E. Benavent and A. Martínez, "Multi-depot Multiple TSP: A polyhedral study and computational results," Annals of Operations Research, vol. 207, no. 1, pp. 7-25, 2013, doi: 10.1007/s10479-011-1024-y.

[2] S. Saud, H. Kodaz, and İ. Babaoğlu, "Solving travelling salesman problem by using optimization algorithms," KnE Social Sciences, vol. 3, no. 1, pp. 17-23, 2018, doi: 10.18502/kss.v3i1.1394.

[3] W. Ho, P. Ji, and P. K. Dey, "A multi-depot travelling salesman problem and its iterative and integrated approaches," International Journal of Operational Research, vol. 1, no. 4, pp. 382-395, 2006, doi: 10.1504/IJOR.2006.010211.

[4] S. Salhi, A. Imran, and N. A. Wassan, "The multi-depot vehicle routing problem with heterogeneous vehicle fleet: Formulation and a variable neighborhood search implementation," Computers \& Operations Research, vol. 52, pp. 315-325, 2014, doi: 10.1016/j.cor.2013.05.011.

[5] X. Xu, Z. Ye, J. Li, and C. Wang, "Solving a large-scale multi-depot vehicle scheduling problem in urban bus systems," Mathematical Problems in Engineering, vol. 2018, 2018, Art. No. 4868906, doi: 10.1155/2018/4868906.

[6] S. Ray, A. Soeanu, J. Berger, and M. Debbabi, "The multi-depot split-delivery vehicle routing problem: Model and solution algorithm," Knowledge-Based Systems, vol. 71, pp. 238-265, 2014, doi: 10.1016/j.knosys.2014.08.006.

[7] S. Nucamendi-Guillén, A. Gómez Padilla, E. Olivares-Benitez, and J. M. Moreno-Vega, "The multi-depot open location routing problem with a heterogeneous fixed fleet," Expert Systems with Applications, vol. 165, 2021, Art. no. 113846, doi: 10.1016/j.eswa.2020.113846.

[8] Y. Chan and S. F. Baker, "The multiple depot, multiple traveling salesmen facility-location problem: Vehicle range, service frequency, and heuristic implementations," Mathematical and Computer Modelling, vol. 41, no. 8-9, pp. 1035-1053, 2005, doi: 10.1016/j.mcm.2003.08.011.

[9] H. Larki and M. Yousefikhoshbakht, "Solving the multiple traveling salesman problem by a novel meta-heuristic algorithm," Journal of Optimization in Industrial Engineering, vol. 16, pp. 55-63, 2014

[10] M. S. Hou and D. B. Liu, "A novel method for solving the multiple traveling salesmen problem with multiple depots," Chinese Science Bulletin, vol. 57, no. 15, pp. 1886-1892, 2012, doi: 10.1007/s11434-012-5162-7.

[11] N. Javadian, F. Adbesh, and K. Kardel, "A new approach to solving the fixed destination multi-depot multiple traveling salesman problem using genetic algorithms," 7th International Conference on Optimization: Techniques and Applications (ICOTA7), 2007, vol. 49, doi: 10.13140/RG.2.1.3958.2244

[12] L. Kota and K. Jármai, "Preliminary studies on the fixed destination mmtsp solved by discrete firefly algorithm," Advanced Logistic systems, vol. 7, no. 2, pp. 95-102, 2013

[13] S. Ghafurian and N. Javadian, "An ant colony algorithm for solving fixed destination multi-depot multiple traveling salesmen problems," Applied Soft Computing, vol. 11, no. 1, pp. 1256-1262, 2011, doi: 10.1016/j.asoc.2010.03.002.

[14] T. Ramadhani, G. F. Hertono, and B. D. Handari, "An ant colony optimization algorithm for solving the fixed destination multi-depot multiple traveling salesman problem with non-random parameters," AIP Conference Proceeding, vol. 1862, no. 1, 2017, Art. No. 030123, doi: 10.1063/1.4991227.

[15] M. Dorigo, V. Maniezzo, and A. Colorni, "Ant system: Optimization by a colony of cooperating agents," IEEE Transactions on Systems, Man, and Cybernetics, Part B (Cybernetics), vol. 26, no. 1, pp. 29-41, 1996, doi: $10.1109 / 3477.484436$

[16] S. Ebadinezhad, "DEACO: Adopting dynamic evaporation strategy to enhance ACO algorithm for the traveling salesman problem," Engineering Applications of Artificial Intelligence, vol. 92, 2020, Art. No. 103649, doi: 10.1016/j.engappai.2020.103649.

[17] K. H. Hingrajiya, R. K. Gupta G. S. Chandel, and G. P. Vishwavidyalaya, "An Approach for Solving Multiple Travelling Salesman Problem using Ant Colony Optimization," Computer Engineering and Intelligent Systems, vol. 6, no. 2, pp. 13-17, 2015

[18] V. Selvi and R. Umarani, "Comparative analysis of ant colony and particle swarm optimization techniques," International Journal of Computer Applications, vol. 5, no. 4, pp. 1-6, 2010. 
[19] X.-S. Yang, Nature-inspired metaheuristic algorithms: Second Edition, Luniver Press, 2010.

[20] I. Fister, X. S. Yang, and J. Brest, "A comprehensive review of firefly algorithms," Swarm and Evolutionary Computation, vol. 13, pp. 34-46, 2013, doi: 10.1016/j.swevo.2013.06.001.

[21] M. Gupta and D. Gupta, "A new modified firefly algorithm," International Journal of Recent Contributions from Engineering, Science \& IT (iJES), vol. 4, no. 2, pp. 18-23, 2016, doi: 10.3991/ijes.v4i2.5879.

[22] M. Younes, "A novel hybrid FFA-ACO algorithm for economic power dispatch," Control Engineering and Applied Informatics, vol. 15, no. 2, pp. 67-77, 2013.

[23] O. I. R. Farisi, B. Setiyono, and R. I. Danandjojo, "A hybrid firefly algorithm - ant colony optimization for traveling salesman problem," Jurnal Buana Informatika, vol. 7, no. 1, pp. 55-64, 2016, doi: 10.24002/jbi.v7i1.484.

[24] A. A. El-Sawy, E. M. Zaki, and R. M. Rizk-Allah, "A novel hybrid ant colony optimization and firefly algorithm for solving constrained engineering design problems," Journal of Natural Sciences and Mathematics, vol. 6, no. 1, pp. 1-22, 2013, doi: 10.12816/0009416.

[25] G. K. Jati and Suyanto, "Evolutionary discrete firefly algorithm for travelling salesman problem," International Conference on Adaptive and Intelligent Systems-ICAIS 2011, vol. 6943, 2011, pp. 393-403, doi: 10.1007/978-3642-23857-4_38.

[26] G. K. Jati, R. Manurung, and Suyanto, "Discrete firefly algorithm for traveling salesman problem: A new movement scheme," Swarm Intelligence and Bio-Inspired Computation, 2013, pp. 295-312, doi: 10.1016/B978-012-405163-8.00013-2

[27] I. Kara and T. Bektas, "Integer linear programming formulations of multiple salesman problems and its variations," European Journal of Operational Research, vol. 174, no. 3, pp. 1449-1458, 2006, doi: 10.1016/j.ejor.2005.03.008.

\section{BIOGRAPHIES OF AUTHORS}
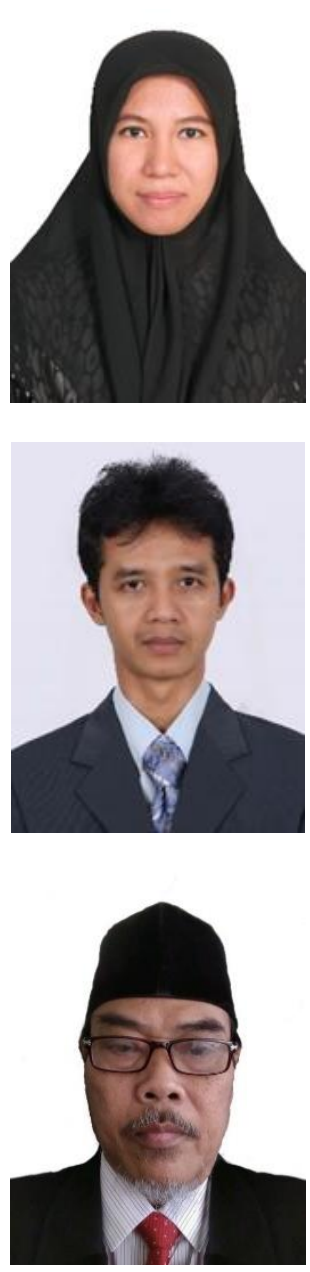

Olief Ilmandira Ratu Farisi is a lecturer at Department of Mathematics Education University of Nurul Jadid (ID). She received a master's degree majoring in Mathematics at Institut Teknologi Sepuluh Nopember (ID) and a bachelor's degree at Mathematics Education State University of Malang (ID). Her research interest are in optimization, graph theory, and development of instructional media.
Budi Setiyono, received the B.Sc. degree in 1997 in mathematics from the Institut Teknologi Sepuluh Nopember (ID), the M.Sc. degree in 2002, in Informatics from the Bandung Institute of Technology (ID) and PhD degree in 2012 from the Institut Teknologi Sepuluh Nopember (ID). He is currently an Assistant Professor in the Institut Teknologi Sepuluh Nopember (ID). His research interests are in Image and video processing, Databases and web technology.

R. Imbang Danandjojo, hold the bachelor degree (1982-1987) in mathematics from Institut Teknologi Sepuluh Nopember (ID). He received the master degree (1995-1997) and doctoral degree (2008-2014) in urban and regional planning from Institut Teknologi Bandung (ID). From 1994 until 2017, he was a researcher at Research and Development Agency of Sea, River, Lake, and Ferry Transportation, Ministry of Transportation Republic of Indonesia. He is currently a lecturer at Politeknik Pelayaran Surabaya (ID). 